\title{
HIV-1 evades innate immune recognition through specific cofactor recruitment
}

\author{
Jane Rasaiyaah ${ }^{1}$, Choon Ping Tan ${ }^{1}$, Adam J. Fletcher ${ }^{1}$, Amanda J. Price ${ }^{2}$, Caroline Blondeau ${ }^{1}$, Laura Hilditch ${ }^{1}$, David A. Jacques ${ }^{2}$, \\ David L. Selwood ${ }^{3}$, Leo C. James ${ }^{2}$, Mahdad Noursadeghi ${ }^{1 *} \&$ Greg J. Towers ${ }^{1 *}$
}

Human immunodeficiency virus (HIV)-1 is able to replicate in primary human macrophages without stimulating innate immunity despite reverse transcription of genomic RNA into doublestranded DNA, an activity that might be expected to trigger innate pattern recognition receptors. We reasoned that if correctly orchestrated HIV-1 uncoating and nuclear entry is important for evasion of innate sensors then manipulation of specific interactions between HIV-1 capsid and host factors that putatively regulate these processes should trigger pattern recognition receptors and stimulate type 1 interferon (IFN) secretion. Here we show that HIV-1 capsid mutants N74D and P90A, which are impaired for interaction with cofactors cleavage and polyadenylation specificity factor subunit 6 (CPSF6) and cyclophilins (Nup358 and CypA), respectively ${ }^{1,2}$, cannot replicate in primary human monocyte-derived macrophages because they trigger innate sensors leading to nuclear translocation of NF-KB and IRF3, the production of soluble type 1 IFN and induction of an antiviral state. Depletion of CPSF6 with short hairpin RNA expression allows wild-type virus to trigger innate sensors and IFN production. In each case, suppressed replication is rescued by IFN-receptor blockade, demonstrating a role for IFN in restriction. IFN production is dependent on viral reverse transcription but not integration, indicating that a viral reverse transcription product comprises the HIV-1 pathogen-associated molecular pattern. Finally, we show that we can pharmacologically induce wild-type HIV-1 infection to stimulate IFN secretion and an antiviral state using a non-immunosuppressive cyclosporine analogue. We conclude that HIV-1 has evolved to use CPSF6 and cyclophilins to cloak its replication, allowing evasion of innate immune sensors and induction of a cell-autonomous innate immune response in primary human macrophages.

HIV-1 capsid (CA) mutant N74D cannot recruit CPSF6 and is insensitive to depletion of HIV-1 cofactors Nup358 and TNPO3, suggesting that it may use alternate cofactors for nuclear entry ${ }^{1-3}$. Furthermore, unlike wild-type (WT) HIV-1, HIV-1 N74D cannot replicate in monocytederived macrophages (MDM) (Fig. 1a and Extended Data Fig. 2) 2,4. Remarkably, an inability to replicate was accompanied by a burst of IFN- $\beta$ detected 2-5 days after low-multiplicity infection (Fig. $1 \mathrm{~b}$ and Extended Data Fig. 2). The antiviral activity of IFN- $\beta$ (Extended Data Fig. $3 a)^{5}$ was revealed by rescuing HIV-1 N74D, but not WT replication with antibody to the IFN- $\alpha / \beta$ receptor $\alpha$ chain (IFNAR2) (Fig. $1 c$, $\mathrm{d}$ and Extended Data Fig. 3b). Co-infection of MDM with WT and HIV-1 N74D led to suppression of WT replication (Fig. 1e), which was also rescued by IFNAR2 antibody (Extended Data Fig. 3c). This demonstrated that sensitivity to IFN-mediated restriction was not limited to the mutant virus.

In contrast to the spreading infection assay in which HIV-1 N74D was completely suppressed, assessment of single-round infection in MDM with higher dose viral inocula revealed only a 5-fold reduction

of HIV-1 N74D infectivity compared to WT (Fig. 1f). However, this reduction was also restored to WT levels by IFNAR2 blockade (Extended Data Fig. 3d). In this experiment we did not detect IFN- $\beta$, probably owing to assay sensitivity, but interferon-stimulated genes (ISGs) IP10 (also known as CXCL10), IFIT1 and CCL8, were induced following infection with HIV-1 N74D, but not WT virus (Extended Data Fig. 4a). ISG induction was confirmed by microarray transcriptional profiling of host responses to HIV-1 N74D, which showed expected enrichment for innate immune type-1 IFN pathways at a genome-wide level (Extended Data Fig. 4c, d). These findings, together with the time course of IFN release during spreading infection (Fig. 1b), indicate that multipleround replication amplifies virus-induced innate responses, leading to high levels of IFN- $\beta$ secretion and potent suppression of HIV-1 replication (Fig. 1a).

We next measured ISG induction in HIV-1 N74D-infected MDM when either DNA synthesis or integration were prevented by mutation of reverse transcriptase ${ }^{6}$ (RT) or integrase $\mathrm{e}^{7}$ (IN), respectively. Infection by HIV-1 CA(N74D), RT(D185E) double mutant did not stimulate IP10 expression, whereas infection with HIV-1 double-mutant CA(N74D), IN(D116N) induced IP10 expression comparable to the WT virus (Fig. 1g). These data indicate that the innate immune response in MDM depends on detection of the products of reverse transcription, not integration.
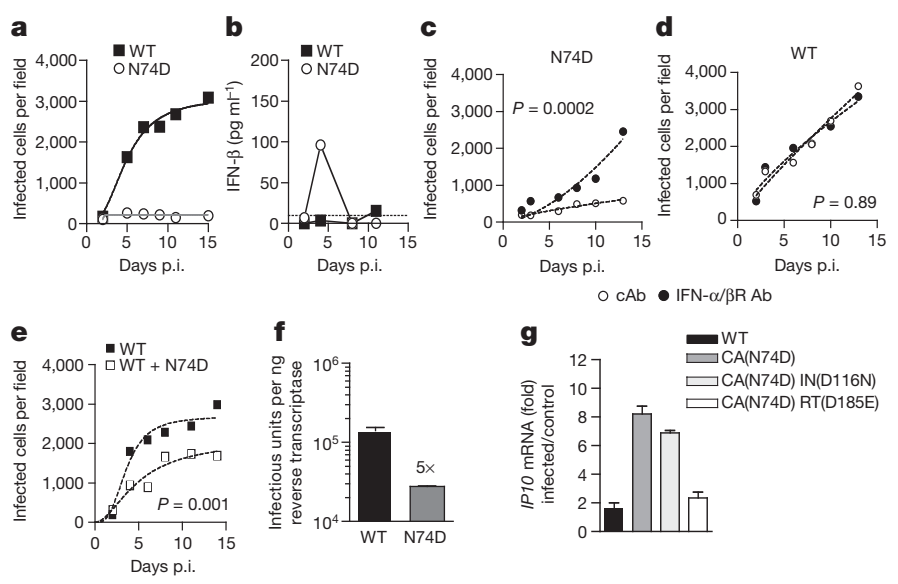

$\circ \mathrm{cAb} \bullet \mathrm{IFN}-\alpha / \beta \mathrm{R} A \mathrm{~b}$
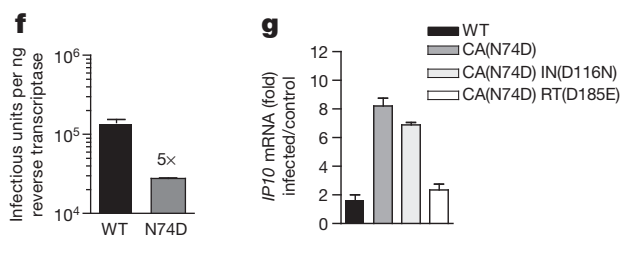

Figure $1 \mid$ HIV-1 CPSF6 binding mutant CA N74D is restricted in MDM due to induction of type 1 IFN. a, Replication of WT HIV-1 or CA mutant N74D in MDM. b, IFN- $\beta$ levels in supernatants from a. c, d, Replication of HIV-1 CA N74D or WT HIV-1 with IFNAR2 or control antibody (cAb). e, Replication of WT or WT plus CA N74D. Mean data and regression lines for biological replicates are shown in c-e. $P$ values (two-way ANOVA) are given for IFNAR2 blockade (c-d) and co-infection with CA mutant N74D (e). f, Infection of MDM by HIV-1 measured at $48 \mathrm{~h}$. g, GAPDH-normalized IP10 mRNA levels expressed as fold change over untreated cells after infection with WT or HIV-1 mutants (mean of 3 technical replicates \pm s.e.m., $\mathbf{f}, \mathbf{g}$ ).

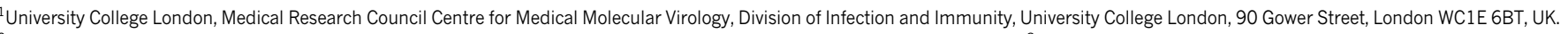

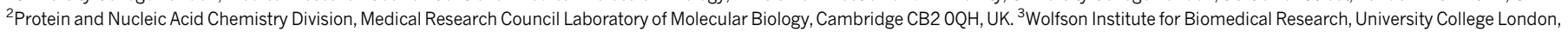
Gower Street, London WC1E 6BT, UK
}

*These authors contributed equally to this work 
Given that CA mutation N74D prevents recruitment of CPSF ${ }^{1,3}$ we proposed that CPSF6 depletion would induce WT HIV-1 to trigger IFN responses in MDM. In fact, CPSF6 depletion by shRNA expression in MDM (Fig. 2a, b and Extended Data Fig. 2) completely abrogated HIV-1 replication (Fig. $2 c$ ) due to a burst of IFN- $\beta$. MDM expressing a non-targeting shRNA did not produce IFN- $\beta$ on HIV-1 infection (Fig. $2 \mathrm{~d}$ ). The restrictive role of IFN was confirmed by rescue of infectivity with IFNAR2 antibody (Fig. 2e). Neither the IFNAR2 nor isotype control antibody had any effect on $\mathrm{HIV}-1$ replication in control shRNA expressing MDM (Fig. 2f). Importantly, shRNA expression itself did not induce IFN- $\beta$ production (Fig. $2 \mathrm{~g}$ ). We conclude that the defect in WT HIV-1 replication after CPSF6 depletion in MDM was largely due to type 1 IFN production. In line with observations made with HIV-1 N74D, CPSF6 depletion also reduced single-round infectivity in MDM by a few fold, 3.5-fold versus 5-fold (Fig. 2h).

The HIV-1 inhibitor PF-3450074 (PF74) binds CA and inhibits CPSF6 recruitment and HIV-1 replication ${ }^{1,3,8}$. As expected, PF74 completely blocked HIV-1 replication in MDM but did not induce soluble IFN- $\beta$ secretion, nor was replication rescued by IFNAR2 blockade (Extended Data Fig. 5a-c). However, as reported, PF74 completely abrogated HIV-1 DNA synthesis (Extended Data Fig. 5d, e) ${ }^{8,9}$. The fact that PF74 mimics CPSF6 binding to HIV-1 CA ${ }^{3}$ suggests that CPSF6 recruitment might prevent premature reverse transcription and innate recognition of viral DNA. To test this hypothesis, a human CPSF6 mutant deleted for its nuclear localization signal (CPSF6 $\Delta \mathrm{NLS})^{10}$, was expressed in HeLa cells. Like PF74, human CPSF6 $\triangle$ NLS blocked VSVG HIV-1 GFP DNA synthesis and infectivity (Extended Data Fig. 5f, g). A CPSF6 mediated block to HIV-1 RT differs from previous observations showing no effect of CPSF6 $\triangle$ NLS on HIV-1 RT but earlier work
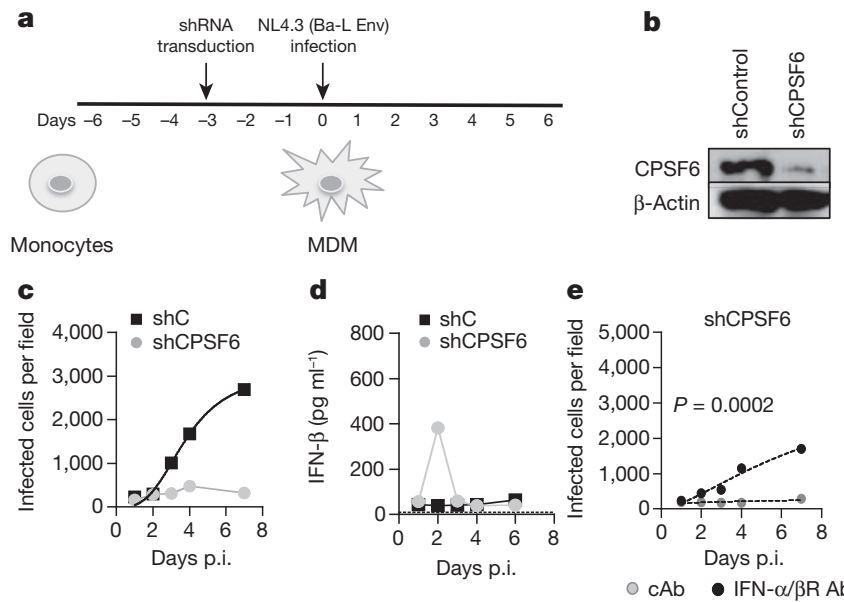

$\mathbf{f}$
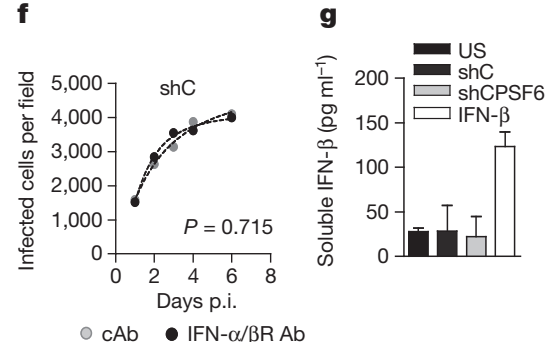

h

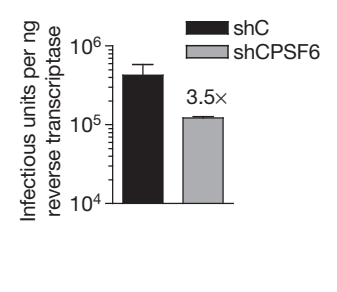

Figure 2 HIV-1 elicits a type 1 IFN response that restricts replication in CPSF6 depleted MDM. a, Protocol schema. b, CPSF6/actin detected at time of infection. c, HIV-1 replication in MDM expressing shRNA targeting CPSF6 or control shRNA. d, IFN- $\beta$ levels in supernatants from $\mathbf{c}$. e, f, Infection of CPSF6depleted or MDM expressing control shRNA with IFNAR2 or control antibody (cAb). $P$ values (two-way ANOVA) are given for the effect of CPSF6 depletion (e) or control shRNA (f) on biological replicates. $\mathbf{g}$, IFN- $\beta$ produced from shRNA-expressing MDM or IFN- $\beta$-treated MDM. h, Infection of MDM by HIV-1 measured at $48 \mathrm{~h}$ on CPSF6-depleted or control shRNA-expressing MDM (mean of 3 technical replicates \pm s.e.m.). used a mouse CPSF6 cDNA with an alternate exon structure ${ }^{1,4}$. We hypothesize that HIV-1 has evolved to recruit CPSF6 to incoming HIV-1 CA and prevent premature DNA synthesis, which would otherwise trigger innate sensors (Extended Data Fig. 1).

Because HIV-1 N74D is unable to appropriately use nuclear pore components and has retargeted integration properties ${ }^{1-3,11}$, we proposed that the HIV-1 CA mutant P90A, which fails to interact with the cyclophilins CypA and nuclear pore component Nup358, and also has retargeted integration ${ }^{2}$, might also trigger innate sensors. Indeed, HIV-1 P90A infection of MDM induced IFN- $\beta$ production and an antiviral state in both replication and single-round infectivity assays, which was rescued by IFN receptor antibody (Fig. 3a-f and Extended Data Figs 2 and 3c, d). We find MDM infection by HIV-1 N74D, P90A or WT were equally increased by macaque simian immunodeficiency virus-like particles (SIVmac VLP) encoding Vpx, indicating that mutant viruses were not specifically Vpx-sensitive (Extended Data Fig. 5h). Quantitative PCR with reverse transcription (qRT-PCR) and wholegenome profiling demonstrated ISG induction after HIV-1 P90A infection (Extended Data Fig. 4b-d). Consistently, double mutation of P90A and RT D185E, but not IN D116N, suppressed IP10 induction (Fig. 3g). We propose that viral DNA produced by reverse transcription is the target for innate sensing of both HIV-1 CA mutants N74D and P90A in MDM.

We next considered the mechanism of HIV-1 mutant innate sensing. A recently identified cytosolic DNA sensor, cyclic GMP-AMP synthase (cGAS), which synthesizes the novel second messenger cGAMP ${ }^{12}$, has been shown to detect HIV-1 reverse-transcribed DNA in human myeloid cells ${ }^{13}$. cGAMP production is detected by stimulator of interferon genes (STING) that transduce an innate signalling cascade, leading to IRF3 activation and type 1 interferon production ${ }^{13}$. We used a biological assay to test for cGAMP production in MDM infected by HIV-1 N74D and P90A. Consistently, extracts from cells infected with HIV-1 $\mathrm{CA}(\mathrm{P} 90 \mathrm{~A})$ mutant contained a benzonase and heat-resistant component that activated an interferon-sensitive promoter in a STING-dependent way (Extended Data Fig. 6). Commercially prepared cGAMP validated the assay and acted as a positive control. Importantly, RNA purified from cells infected with HIV-1 mutant was not immunostimulatory, as oppose to RNA from cells infected with Sendai virus, which potently activated an IFN- $\beta$ promoter, as expected for a RIG-I-triggering virus.
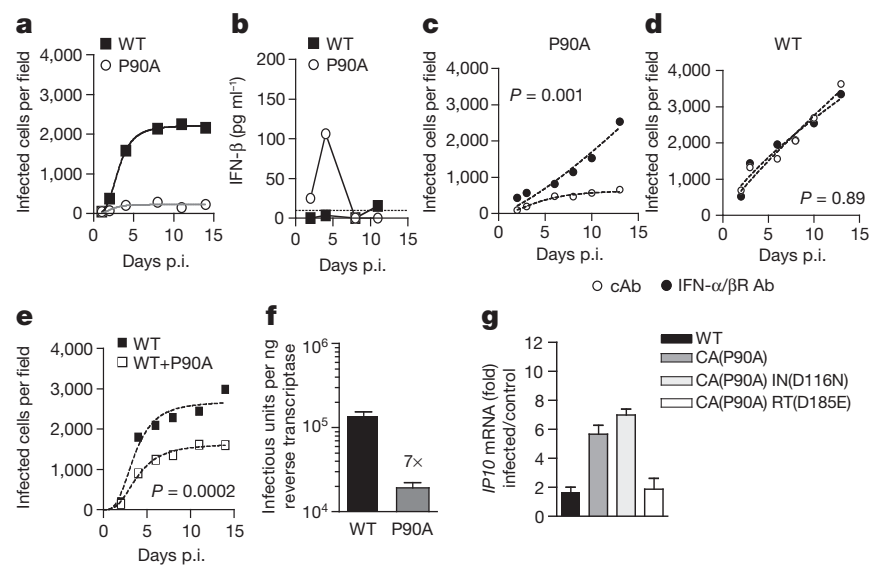

Figure 3 HIV-1 CypA-binding mutant CA(P90A) is restricted in MDM owing to induction of type 1 IFN. a, Replication of WT HIV-1 or CA mutant P90A in MDM. b, IFN- $\beta$ levels in supernatants from a. c, Replication of HIV-1 CA(P90A) with IFNAR2 or control antibody (cAb). d, As in Fig. 1d. e, Replication of WT or WT plus CA(P90A). Mean data and regression lines are shown for biological replicates in c-e. $P$ values (two-way ANOVA) are given for IFNAR2 blockade (c, d) and co-infection with CA mutant P90A (e).f, Infection of MDM by HIV-1 measured at $48 \mathrm{~h}$. g, GAPDH-normalized IP10 mRNA levels expressed as fold change over untreated cells after infection with WT or HIV-1 mutants (mean of 3 technical replicates \pm s.e.m.; $\mathbf{f}, \mathbf{g}$ ). 
These data support our hypothesis that HIV-1 DNA is the pathogenassociated molecular pattern. Intriguingly, HIV-1 N74D did not stimulate in either of these assays, indicating that the two HIV-1 mutants activate independent DNA sensors. This possibility is consistent with the different integration site targeting preferences of the two mutants with HIV-1 N74D and P90A integrating into lower gene density or higher gene density regions of chromatin, respectively, compared to wild-type virus ${ }^{2}$.

Immunofluorescent detection of NF- $\kappa \mathrm{B}$ and IRF3 revealed nuclear translocation of both transcription factors after exposure to either of the HIV-1 mutants but not WT virus (Fig. $4 \mathrm{a}-\mathrm{c}$ and Extended Data Fig. 7). Concordantly, inhibition of NF- $\kappa B$ activation with a peptide inhibitor of NEMO (IKK) rescued infectivity of both HIV-1 mutants in a dose-dependent manner (Fig. 4c). Finally, we considered whether prevention of cofactor interaction using drugs could induce WT virus to trigger a cell-autonomous innate immune response in the same way as mutant virus. We sought to phenocopy the HIV-1 P90A mutant by inhibiting cyclophilin recruitment using cyclosporine or a nonimmunosuppressive analogue of cyclosporine, SmBz-CsA. SmBz-CsA is modified at the $3^{\prime}$-SAR position to include a methylphenyl-4-carboxylic
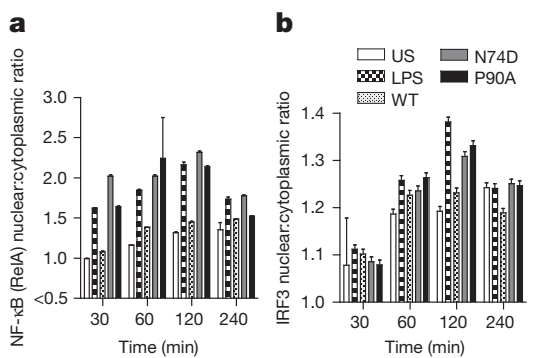

c
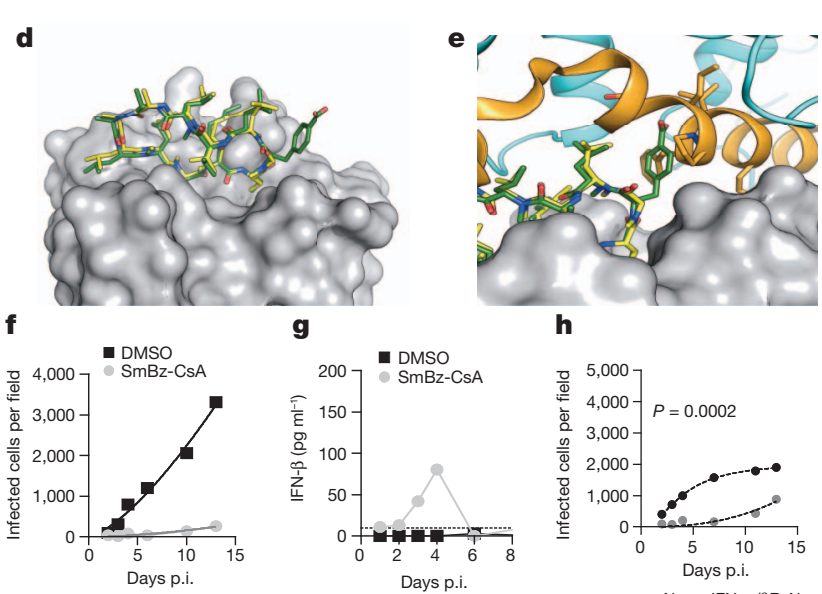

g h
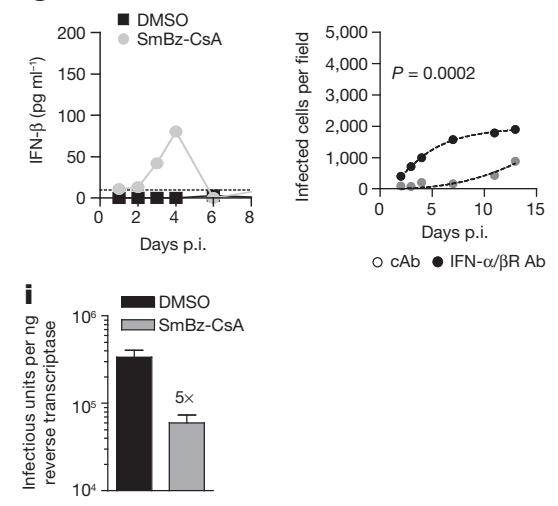

$\circ \mathrm{cAb} \bullet \operatorname{IFN}-\alpha / \beta \mathrm{R} A \mathrm{Ab}$

Figure $4 \mid$ NF-кB/IRF3 are activated by mutant HIV-1 and SmBz-CsA treatment causes WT HIV-1 to trigger innate responses. a, b, Mean

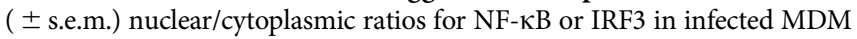
$(P<0.05$, two-way ANOVA)., LPS, lipopolysaccharide; Nemo, inhibitor peptide to IKK- $\alpha / \beta$; US, unstimulated. c, Infection at $48 \mathrm{~h} \pm$ IKK inhibitor. d, e, SmBz-CsA (green) complexed with CypA (grey), cyclosporine (yellow) and calcineurin (orange/blue). $\mathbf{f}$, Replication of HIV-1 in MDM \pm SmBz-CsA; g, IFN- $\beta$ levels from f. h, MDM infected with WT HIV-1 plus SmBz-CsA and IFNAR2 antibody or $\mathrm{CAb}$ (mean data and regression lines). $P$ value (two-way ANOVA) is given for IFNAR2 blockade of biological replicates. i, Infection of MDM by WT HIV $-1 \pm \mathrm{SmBz}-\mathrm{CsA}$ at $48 \mathrm{~h}$ (mean of 3 technical replicates \pm s.e.m.). acid group and therefore cannot inhibit calcineurin or affect $\mathrm{T}$ cell activation $^{14}$ (Fig. 4d, e and Extended Data Table 1). Like cyclosporine, SmBz-CsA inhibited recruitment of CypA, but not Nup358 Cyp, to HIV-1 CA (Extended Data Fig. 8e). Treatment of MDM with SmBzCsA (Fig. 4f) or cyclosporine (Extended Data Fig. 8a) completely suppressed WT HIV-1 replication and elicited IFN- $\beta$ production (Fig. $4 \mathrm{~g}$ and Extended Data Fig. 8b). Inhibited viral replication was rescued by IFNAR2 blockade, but not by control antibody (Fig. 4h and Extended Data Fig. 8c). After single-round infection in the presence of either drug, infection was 5-7-fold lower (Fig. 4i and Extended Data Fig. 8d). These data are consistent with observations of cyclosporine inhibition of hepatitis $C$ virus, in which innate immune responses are implicated $^{15}$.

Our findings demonstrate that human macrophages are able to detect HIV-1 infection and activate a cell-autonomous innate immune signal, when specific interactions with HIV-1 cofactors are prevented by virus mutation (Figs 1 and 3), depletion of cofactor expression (Fig. 2) or pharmacological inhibition of cofactor recruitment (Fig. 4). We envisage that appropriate interaction between CA and CPSF6/cyclophilins normally allows evasion of innate sensors and promotes HIV-1 infection. We propose a model in which CPSF6/CypA recruitment to CA suppresses premature viral DNA synthesis and thus innate triggering. Inhibition of DNA synthesis by CPSF6 $\triangle N L S$ or the CPSF6 mimic PF74 support this possibility. In our model, nuclear entry of CPSF6 could release the virus, therefore enabling reverse transcription at the nuclear pore. The cytosolic exonuclease TREX1 degrades excess cytoplasmic DNA and prevents cGAS activation and IFN stimulation ${ }^{13,16}$ (Extended Data Fig. 8f-j). Our data indicate that DNA synthesized by HIV-1 mutants is insensitive to TREX1 degradation, either through nature or location, and in the case of HIV-1 CA(P90A), is detected by cGAS leading to cGAMP production. In MDDC, CypA has been suggested to have a different role, acting to aid detection of HIV-1 by innate sensors during egress ${ }^{17}$. These observations suggest that HIV1 may rely on cell-type-specific cofactor use to protect it from innate immune defences. Intriguingly, both N74D and P90A CA mutants replicated in indicator cell lines GHOST and HeLa TZM-bl to WT levels (Extended Data Fig. 9). Replication was unaffected by IFNreceptor blockade and ISG expression was not induced, illustrating that these cell lines cannot respond in the same way to HIV-1 infection. They also suggest that the only obstacle to HIV-1 CA mutant replication in MDM is due to induction of innate responses. Our observations facilitate the further study of the relationship between HIV-1 and innate immunity. We envisage therapeutics, or vaccine adjuvants, which induce virus to trigger potent cell-autonomous innate immunity, IFN secretion and enhanced adaptive immune responses.

\section{METHODS SUMMARY}

Monocytes were isolated and incubated with macrophage colony stimulating factor (M-CSF) to induce macrophage differentiation. Full-length HIV-1 from molecular clones and virus-like particles (VLPs) were produced by transient transfection of HEK293T cells and purified by centrifugation through a sucrose cushion. Infections were performed by incubating $10^{5} \mathrm{MDM}$ per well in 48 -well plates. Intracellular staining of p24 was performed at various time points post infection with anti-p24 antibodies followed by a LacZ-conjugated antibody and automated colony counting using an ELISPOT reader (AID). Type 1 interferon was measured by enzyme linked immunosorbent assay (PBL Interferon Source) in supernatants taken from the wells used to measure HIV-1 replication. Single-round HIV-1 infections of MDM were performed by measuring HIV-1 p24-positive cells, as above, $48 \mathrm{~h}$ post infection. In CPSF6 and TREX1 depletion experiments day 3 differentiating MDM were transduced with shRNA encoding HIV-1 vector and SIVmac VLPs encoding Vpx and day 6 cells were challenged with replication competent HIV-1. The cGAMP assay was performed by treating L929 cells stably expressing luciferase under the control of an interferon-sensitive response element with heatand benzonase-treated extracts from infected MDM. Immunostimulatory RNA was assayed by sequentially transfecting $293 \mathrm{~T}$ cells with a luciferase reporter under the control of the interferon beta promoter and immunostimulatory RNA purified from MDM infected with HIV-1, or Sendai virus as a positive control. Nuclear 
translocation of IRF3 and NF- $\mathrm{\kappa B}$ was assessed by staining HIV-1 infected MDM with specific antibodies and measuring nuclear to cytoplasmic staining ratios.

Online Content Any additional Methods, Extended Data display items and Source Data are available in the online version of the paper; references unique to these sections appear only in the online paper.

Received 5 February; accepted 8 October 2013.

Published online 6 November; corrected online 20 November 2013 (see full-text HTML version for details).

1. Lee, K. et al. Flexible use of nuclear import pathways by HIV-1. Cell Host Microbe $\mathbf{7}$, 221-233 (2010).

2. Schaller, T. et al. HIV-1 capsid-cyclophilin interactions determine nuclear import pathway, integration targeting and replication efficiency. PLoS Pathog. 7, e1002439 (2011)

3. Price, A. J. et al. CPSF6 defines a conserved capsid interface that modulates HIV-1 replication. PLoS Pathog. 8, e1002896 (2012).

4. Ambrose, Z. et al. Human immunodeficiency virus type 1 capsid mutation N74D alters cyclophilin A dependence and impairs macrophage infection. J. Virol. 86, 4708-4714 (2012).

5. Tsang, J. et al. HIV-1 infection of macrophages is dependent on evasion of innate immune cellular activation. AIDS 23, 2255-2263 (2009).

6. Besnier, C., Takeuchi, Y.\& Towers, G. Restriction of lentivirus in monkeys. Proc. Nat Acad. Sci. USA 99, 11920-11925 (2002).

7. Iyer, S. R., Yu, D., Biancotto, A., Margolis, L. B. \& Wu, Y. Measurement of human immunodeficiency virus type 1 preintegration transcription by using Revdependent Rev-CEM cells reveals a sizable transcribing DNA population comparable to that from proviral templates. J. Virol. 83, 8662-8673 (2009).

8. Shi, J., Zhou, J., Shah, V. B., Aiken, C. \& Whitby, K. Small-molecule inhibition of human immunodeficiency virus type 1 infection by virus capsid destabilization. J. Virol. 85, 542-549 (2011).

9. Blair, W. S. et al. HIV capsid is a tractable target for small molecule therapeutic intervention. PLoS Pathog. 6, e1001220 (2010).

10. Dettwiler, S., Aringhieri, C. Cardinale, S., Keller, W. \& Barabino, S. M. Distinct sequence motifs within the 68-kDa subunit of cleavage factor $I_{m}$ mediate RNA binding, protein-protein interactions, and subcellular localization. J. Biol. Chem. 279, 35788-35797 (2004)
11. Ocwieja, K. E et al. HIV integration targeting: a pathway involving Transportin-3 and the nuclear pore protein RanBP2. PLoS Pathog. 7, e1001313 (2011).

12. Sun, L., Wu, J., Du, F., Chen, X. \& Chen, Z. J. Cyclic GMP-AMP synthase is a cytosolic DNA sensor that activates the type I interferon pathway. Science 339, 786-791 (2013).

13. Gao, D. et al. Cyclic GMP-AMP synthase is an innate immune sensor of HIV and other retroviruses. Science 341, 903-906 (2013).

14. Dube, H. et al. A mitochondrial-targeted cyclosporin A with high binding affinity for cyclophilin D yields improved cytoprotection of cardiomyocytes. Biochem. J. 441, 901-907 (2012).

15. Liu, J.-P., Ye, L., Wang, X., Li, J.-L. \& Ho, W.-Z. Cyclosporin A inhibits hepatitis C virus replication and restores interferon-alpha expression in hepatocytes. Transpl. Inf. Dis. 13, 24-32 (2011)

16. Yan, N., Regalado-Magdos, A. D., Stiggelbout, B., Lee-Kirsch, M. A. \& Lieberman, J. The cytosolic exonuclease TREX 1 inhibits the innate immune response to human immunodeficiency virus type 1. Nature Immunol. 11, 1005-1013 (2010).

17. Manel, N. et al. A cryptic sensor for HIV-1 activates antiviral innate immunity in dendritic cells. Nature 467, 214-217 (2010).

Acknowledgements We are grateful to J. W. Chin, S. Goodbourn, K. Lee, O. Perisic and V. KewalRamani for reagents and advice. This work was funded by Wellcome Trust Senior Fellowship 090940 to G.J.T., the Medical Research Council, an MRC Confidence in Concept Award to G.J.T. and D.S. and the National Institute for Health Research (NIHR) University College London Hospitals Biomedical Research Centre. The views expressed are those of the authors and not necessarily those of the NHS, the NIHR or the Department of Health.

Author Contributions J.R., C.P.T., A.J.F., D.L.S., M.N. and G.J.T. designed the study. J.R performed all experiments in MDM, C.P.T. performed cGAMP and immunostimulatory RNA assays, A.J.F. performed CPSF6 experiments in HeLa cells. A.J.P. solved the structure of SmBz-CsC in complex with CypA and L.H. performed the TRIMCyp experiments. J.R., A.J.F., A.J.P., L.H., D.A.J., L.C.J., M.N. and G.J.T. analysed the data. A.J.F. and C.B. generated constructs and D.L.S. synthesized SmBz-CsA. J.R., M.N. and G.J.T. wrote the manuscript.

Author Information Structural coordinates have been deposited under PDB accession code 4IPZ. Microarray data are available from the EBI Array Express repository under accession no. E-MTAB-1437. Reprints and permissions information is available at www.nature.com/reprints. The authors declare no competing financial interests. Readers are welcome to comment on the online version of the paper. Correspondence and requests for materials should be addressed to G.J.T. (g.towers@ucl.ac.uk) and M.N. (m.noursadeghi@ucl.ac.uk). 


\section{METHODS}

Cells. Primary monocyte-derived macrophages (MDM) were prepared from fresh blood from healthy volunteers as described ${ }^{5}$. The study was approved by the joint University College London/University College London Hospitals NHS Trust Human Research Ethics Committee and written informed consent was obtained from all participants. Briefly, peripheral blood mononuclear cells were isolated by Ficoll-Hypaque (Axis-Shield) density centrifugation. The isolated cells were washed with PBS and plated in RPMI (Invitrogen) supplemented with 10\% heat-inactivated autologous human serum (HS) and $40 \mathrm{ng} \mathrm{ml}^{-1}$ macrophage colony stimulating factor (M-CSF) (R\&D systems). The medium was then refreshed after 3 days (RPMI 1640 with $10 \% \mathrm{HS}$ ), removing any remaining non-adherent cells. After 6 days, media was replenished with RPMI containing 5\% type AB HS (Sigma-Aldrich). Replicate experiments were performed with cells derived from different donors.

$\mathrm{GHOST}^{18}$, a human osteosarcoma cell line stably expressing CD4, CCR5, CXCR4 and the green fluorescent protein $(G F P)$ reporter gene under the control of the HIV-2 long terminal repeat were maintained in DMEM containing $10 \%$ heatinactivated fetal calf serum (FCS), glutamine, antibiotics, G418 $\left(500 \mu \mathrm{g} \mathrm{ml}^{-1}\right)$, hygromycin $\left(100 \mu \mathrm{g} \mathrm{ml}^{-1}\right)$, and puromycin $\left(1 \mu \mathrm{g} \mathrm{ml}^{-1}\right)$ and were split twice a week. HEK293T cells were grown in DMEM (Invitrogen) supplemented with $10 \%$ FCS

Reagents. Recombinant human interferon (IFN)- $\beta$ (Merck Serono) was used at $10 \mathrm{ng} \mathrm{ml}^{-1}$, poly(I:C) (Sigma) was used at $10 \mu \mathrm{g} \mathrm{ml}^{-1}$, Cyclosporine (Sandoz) was used at $5 \mu \mathrm{M}$. SmBz-CsA was synthesized as described ${ }^{14}$ and used at $10 \mu \mathrm{M}$. PF74, a gift from J. Chin, was synthesized as described ${ }^{3}$ and used at $10 \mu \mathrm{M}$. Lipopolysaccaride (LPS) (Sigma) was used at $100 \mathrm{ng} \mathrm{ml}^{-1}$. Commercially prepared STING agonist, cyclic GMP-AMP (cGAMP), was purchased from InvivoGen.

Plasmids. The CCR5-tropic wild-type NL4.3 (Ba-L Env) or NL4.3 (Ba-L Env) bearing CA mutations P90A or N74D were derived from an infectious clone of NL4.3 by cloning the Env gene from HIV-1 Ba-L between unique EcoR1 and BamH1 sites to replace the NL4.3 Env gene. $\Delta \mathrm{RT}$ and $\Delta \mathrm{IN}$ infectious clones were generated by making mutant $\mathrm{RT}(\mathrm{D} 185 \mathrm{E})^{6}$, or $\mathrm{IN}(\mathrm{D} 116 \mathrm{~N})^{7}$ using site-directed mutagenesis (Stratagene).

Short hairpin sequences were expressed from HIV-1-based shRNA expression vector HIVSiren ${ }^{2}$. CPSF6 shRNA target sequence was $5^{\prime}$-CGAAGAGTTCAACC AGGAA-3'; TREX1 shRNA target sequence was $5^{\prime}$-CCAAGACCATCTGCTG TCA-3'; CPSF6 was detected by western blot and TREX-1 was detected by qRT-PCR.

A human CPSF6 expression vector was prepared by PCR cloning the human CPSF6 ORF from complementary DNA (Superscript, Life Technologies), prepared from HeLa cells, into the MLV based gammaretroviral expression vector $\mathrm{EXN}^{19}$ using primers forward $5^{\prime}$-ATCGGAATTCATGGCGGACGGTGTGGAC CACATAGACATTTAC- $3^{\prime}$ and reverse $5^{\prime}$-ATGCGCGGCCGCCTAACGATG ACGATATTCGCGCTCTC-3', restriction sites underlined. The nuclear localization signal was removed from CPSF6 as described ${ }^{10}$ by deleting the C-terminal 50 amino acids by PCR using reverse primer 5 '-ATGCGCGGCCGCTCATTCT CGTGATCTACTATGGTCCC-3' and forward primer as above. The resulting NLS mutant is defective for nuclear entry as described ${ }^{10}$. CPSF $6 \Delta$ NLS was expressed in HeLa cells by gammaretroviral vector transduction as described ${ }^{3}$ and G418selected pools of cells generated. Note that the human CPSF6 cDNA described herein differs from the murine cDNA described previously ${ }^{1,4,20}$ in that it represents the most common human CPSF6 isoform represented by GenBank accession number nm007007 and thus lacks exon $6^{21-23}$.

Virus production. Virus particles were produced by transient transfection of HEK293T cells. $3.5 \mu \mathrm{g}$ of molecular clone DNA; for shRNA we used $1.5 \mu \mathrm{g}$ pHIVSIREN $^{2}$ shRNA, $1 \mu \mathrm{g}$ p $8.91^{24}$ and $1 \mu \mathrm{g}$ pMDG $^{25}$ encoding VSV-G protein. For SIVmac-VLP we left out the genome plasmid and transfected $3 \mu \mathrm{g}$ pSIV $3+{ }^{26}$ and $1 \mu \mathrm{g}$ pMDG using $10 \mu \mathrm{l}$ FuGENE 6 transfection reagent (Promega) as described $^{6}$. HIV-1 GFP was produced by transfection of 293 T with GFP-encoding genome CSGW, packaging plasmid p8.91 and pMDG as described ${ }^{6}$. Virus supernatants were collected $48 \mathrm{~h}, 72 \mathrm{~h}$ and $96 \mathrm{~h}$ post transfection. All virus suspensions were filtered and ultracentrifuged through a $20 \%$ sucrose buffer and resuspended in RPMI 1640 with 5\% HS, for subsequent infection of MDM. All virus preparations were quantified by reverse transcriptase (RT) enzyme-linked immunosorbent assay (ELISA) (Roche) except when doses were measured by p24 CA ELISA (National Cancer Institute at Frederick) where stated (Figs 1g and 3g). Viruses were also titrated on GHOST where described detecting infection by flow cytometry $72 \mathrm{~h}$ post infection or HeLa TZM bl where infection was detected by CA staining as below.

Infection and stimulation. MDM were infected with $100 \mathrm{pg}$ reverse transcriptase enzyme-linked immunosorbent assay (RT-ELISA) (Roche) per well (multiplicity of infection (MOI) 0.2) in 48-well plates and subsequently fixed and stained using CA-specific antibodies (EVA365 and EVA366 National Institute of Biological Standards AIDS Reagents Programme) and a secondary antibody linked to beta galactosidase, as described ${ }^{5}$. During the time course, supernatants were collected for IFN- $\beta$ ELISA (PBL Interferon Source) according to manufacturer's instructions. Anti-IFN- $\alpha / \beta$ receptor (PBL Interferon Source) or control IgG2A antibody (R\&D systems) were added at $1 \mu \mathrm{g} \mathrm{ml}^{-1}$ for $2 \mathrm{~h}$ before infection and supplemented every 4 days. For inhibition of NF- $\mathrm{\kappa B}$ activation, a peptide inhibitor of NEMO (IKK $\gamma$ ) or control peptide (Imgenex), were added at either $50 \mu \mathrm{M}$ or $100 \mu \mathrm{M}$ for $12 \mathrm{~h}$ before infection. For Agilent microarray analysis and qRT-PCR, MDM were infected with $1 \mathrm{ng}$ RT per well (MOI 2) in 24-well plates. RNA was extracted $24 \mathrm{~h}$ post infection (RNeasy, Qiagen) and subject to microarray analysis as described later. For shRNA transduction of MDM, day 3 differentiated cells were infected with shRNA (0.1 ng RT per ml), SIVmac-VLP (1 ng RT per ml) $+8 \mu \mathrm{g} \mathrm{ml}^{-1}$ polybrene overnight.

Western blot analysis. CPSF6 expression was measured in extracted cell pellets by western blot. Cells were lysed in Laemmli buffer then boiled before separation by SDS-PAGE as described previously ${ }^{3}$. After CPSF6 or STING detection membranes were stripped and probed again for $\beta$-actin as a loading control. Antibodies used were CPSF6 (Abcam ab99347) STING (Abcam ab82960) and $\beta$-actin (Abcam ab6276).

Microarray analysis. Total RNA was purified from cell lysates collected in RLT buffer (Qiagen) using the RNeasy Mini kit (Qiagen). Samples were processed for Agilent microarrays as previously described ${ }^{5}$ and loess normalized data were analysed using the TM4 microarray software suite $\mathrm{MeV} v 4.8^{27}$. Pathway enrichment analysis of differentially expressed gene lists was performed using the online bioinformatics tool InnateDB ${ }^{28}$. Microarray data are available from the EBI Array Express repository (http://www.ebi.ac.uk/arrayexpress/) under accession no EMTAB-1437.

Quantitative PCR. cDNA was synthesized using the Omniscript RT Kit (Qiagen) and quantitative PCR of selected genes was performed using the following inventoried TaqMan assays (Applied Biosystems) CCL8 (Hs04187715_m1) and IFIT1 (Hs01911452_s1). IP10 expression was quantified using: forward primer: $5^{\prime}$-TGA AATTATTCCTGCAAGCCAATT-3' , reverse primer: 5'-CAGACATCTCTTCT CACCCTTCTTT- ${ }^{\prime}$, and probe: $5^{\prime}$-TGTCCACGTGTTGAGATCATTGCTACA ATG-3'. TREX1 expression was quantified using: forward primer: $5^{\prime}$-GCATCTG TCAGTGGAGACCA-3' ${ }^{\prime}$, reverse primer: $5^{\prime}$-AGATCCTTGGTACCCCTGCT- ${ }^{\prime}$, and probe: 5'-CACAACCAGGAACACTAGTCCCAGC-3'. Expression levels of target genes were normalized to glyceraldehyde-3-phosphate dehydrogenase $(G A P D H)$ as previously described ${ }^{5}$. To measure late reverse transcription products, total DNA was purified $9 \mathrm{~h}$ post infection (QIAamp, Qiagen) with DNasetreated virus ( $70 \mathrm{U} \mathrm{ml}^{-1}$ DNase (Affymetrix) in RQ1 buffer (Promega) for $37^{\circ} \mathrm{C}$ $1 \mathrm{~h}$ ) and $500 \mathrm{ng}$ were subjected to TaqMan quantitative PCR using late reverse transcription primers and probe to detect provirus as described ${ }^{29}$. Cells were infected with virus that had been boiled for $2 \mathrm{~min}$ as a negative control. Infectivity was measured in parallel samples by intracellular p 24 staining $48 \mathrm{~h}$ post infection. Presented qPCR experiments are means of technical replicates and represent 3 biological replicates.

cGAMP reporter assay. MDM were infected with $1 \mathrm{ng} \mathrm{RT}$ per well (MOI 2) in 24 well plates for $18 \mathrm{~h}$. Cells were lysed in hypotonic buffer $(10 \mathrm{mM}$ Tris $\mathrm{pH} 7.4$, $10 \mathrm{mM} \mathrm{KCl}$ and $1.5 \mathrm{mM} \mathrm{MgCl}_{2}$ ). After freeze thaw, a proportion of cell extract was kept for stimulatory RNA reporter assay and the remaining was heated to $96^{\circ} \mathrm{C}$ for $10 \mathrm{~min}$. Sonicated extracts were centrifuged $\left(20,000 \mathrm{~g}, 20 \mathrm{~min}, 4^{\circ} \mathrm{C}\right)$,

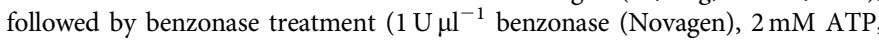
$37^{\circ} \mathrm{C} 90 \mathrm{~min}$ ). $4 \mu \mathrm{l}$ of lysate was introduced to reporter cells using Lipofectamine 2000 (Invitrogen). The reporter cells are L929 cells or L929 cells depleted of STING by transfecting (Oligofectamine, Invitrogen) a previously described STING siRNA ${ }^{30}$. The L929 cells stably express firefly luciferase driven by an interferon sensitive response element. Luciferase was read after $16 \mathrm{~h}$ using Steady-Glo (Promega) and a luminometer

Immunostimulatory RNA reporter assay. Cell extracts were subjected to TRIzol extraction (Invitrogen) and the extracted RNA, plus a control plasmid encoding Renilla luciferase, was transfected into 293T cells expressing firefly luciferase driven by IFN- $\beta$ promoter. Cells were transfected with $500 \mathrm{ng}$ of RNA extracted from macrophages and luciferase values were determined at $16 \mathrm{~h}$ using dualluciferase assay kit (Promega). IFN- $\beta$ promoter activity (firefly luciferase) was normalized by global transcription (Renilla luciferase) and fold induction compares normalized luciferase values against mock-transfected reporter cells. As a positive control we infected MDM with Sendai virus, a gift from S. Goodbourn, and purified immunostimulatory RNA. All transfections in this assay use Lipofectamine 2000 (Invitrogen).

Quantitative confocal immunofluorescence analysis of NF- $\mathrm{BB}$ and IRF3 nuclear translocation. Nuclear/cytoplasmic ratios of NF- $\kappa B$ RelA and IRF3 transcription factors were analysed as previously described ${ }^{5}$ using a Hermes WiScan Cell Imaging System to analyse cells stained with rabbit polyclonal anti NF- $\kappa B$ 
RelA (clone C-20) (Santa Cruz Biotechnology) or rabbit polyclonal anti IRF3 (clone FL 425) (Santa Cruz Biotechnology).

Protein expression, purification, crystallization, data collection, structure determination and refinement. CypA was expressed in Escherichia coli C41(DE3) cells (Lucigen) from tagless expression vector pOPT ${ }^{3}$. Cells were grown overnight at $18^{\circ} \mathrm{C}$ before being collected, sonicated and purified by SP ion-exchange chromatography (GE Healthcare) followed by gel filtration. Crystals of SmBz-CsA in complex with CypA were grown at $17^{\circ} \mathrm{C}$ in sitting drops. Protein solution $(1 \mathrm{mM}$ each of CypA and SmBz-CsA in $20 \mathrm{mM}$ Tris pH 8, $50 \mathrm{mM} \mathrm{NaCl}, 1 \mathrm{mM}$ DTT, $1 \%$ DMSO) was mixed with reservoir solution ( $1 \mathrm{M} \mathrm{LiCl}, 0.1 \mathrm{M} \mathrm{MES} \mathrm{pH} 6,30 \% \mathrm{w} / \mathrm{v}$ PEG 6000) in a 1:1 mix, producing $0.15 \mathrm{~mm} \times 0.10 \mathrm{~mm} \times 0.10 \mathrm{~mm}$ crystals within $24 \mathrm{~h}$. Crystals were flash-frozen in liquid nitrogen before data collection using an in-house Mar-345 detector. Crystal data and diffraction statistics are provided in Extended Data Table 1. Crystallographic analysis was performed using programs from the CCP4 suite ${ }^{31}$. Data were indexed and scaled in MOSFLM and SCALA, respectively. The structure of SmBz-CsA:CypA (pdb 1CWA ${ }^{32}$ ) was used as a search model. Structures were refined in REFMAC and $\mathrm{Coot}^{31}$. Structural figures were created using PyMol (http://pymol.sourceforge.net/). PDB coordinates have been deposited under accession code 4IPZ.

Statistical methods. All data were normally distributed and analysed for statistically significant differences between experimental groups by $t$-tests or two-way ANOVA as indicated. Bar charts show mean \pm s.e.m. for experimental replicates in each case. Replication assays are presented for individual experiments, or where $P$ values (two-way ANOVA) are given, replicate experiments. Individual or mean data points and nonlinear regression lines are shown over time. Sample sizes for each experiment were based on pilot experiments to estimate the effect size and variance of the data.

18. Mörner, A. et al. Primary human immunodeficiency virus type 2 (HIV-2) isolates, like HIV-1 isolates, frequently use CCR 5 but show promiscuity in coreceptor usage. J. Virol. 73, 2343-2349 (1999).
19. Zhang, F, Hatziioannou, T, Perez-Caballero, D, Derse, D. \& Bieniasz, P. D. Antiretroviral potential of human tripartite motif- 5 and related proteins. Virology 353, 396-409 (2006).

20. Lee, K. et al. HIV-1 capsid-targeting domain of cleavage and polyadenylation specificity factor 6. J. Virol. 86, 3851-3860 (2012).

21. Ruepp, M. D., Schumperli, D. \& Barabino, S. M. mRNA3' end processing and moremultiple functions of mammalian cleavage factor I-68. Wiley interdisciplinary reviews. RNA 2, 79-91 (2011)

22. Yang, Q., Gilmartin, G. M. \& Doublie, S. The structure of human cleavage factor $I_{m}$ hints at functions beyond UGUA-specific RNA binding: a role in alternative polyadenylation and a potential link to $5^{\prime}$ capping and splicing. RNA Biol. 8, 748-753 (2011).

23. Hori, T. et al. A carboxy-terminally truncated human CPSF6 lacking residues encoded by exon 6 inhibits HIV-1 cDNA synthesis and promotes capsid disassembly. J. Virol. 87, 7726-7736 (2013)

24. Zufferey, R., Nagy, D., Mandel, R. J., Naldini, L. \& Trono, D. Multiply attenuated lentiviral vector achieves efficient gene delivery in vivo. Nature Biotechnol. 15, 871-875 (1997)

25. Naldini, L. et al. In vivo gene delivery and stable transduction of non-dividing cells by a lentiviral vector. Science 272, 263-267 (1996).

26. Nègre, D. et al. Characterization of novel safe lentiviral vectors derived from simian immunodeficiency virus (SIVmac251) that efficiently transduce mature human dendritic cells. Gene Ther. 7, 1613-1623 (2000)

27. Saeed,A. I. et al. TM4: a free, open-source system for microarray data management and analysis. Biotechniques 34, 374-378 (2003).

28. Lynn, D. J. et al. Curating the innate immunity interactome. BMC Syst. Biol. 4, 117 (2010).

29. Butler, S. L., Hansen, M. S. \& Bushman, F. D. A quantitative assay for HIV DNA integration in vivo. Nature Med. 7, 631-634 (2001)

30. Ishikawa, H, Ma, Z \& Barber, G. N. STING regulates intracellular DNA-mediated, type I interferon-dependent innate immunity. Nature 461, 788-792 (2009).

31. Collaborative Computational Project, number 4 . The CCP4 suite: programs for protein crystallography. Acta Crystallogr. D 50, 760-763 (1994).

32. Mikol, V., Kallen, J., Pflugl, G. \& Walkinshaw, M. D. X-ray structure of a monomeric cyclophilin A-cyclosporin A crystal complex at 2.1 A resolution. J. Mol. Biol. 234, 1119-1130 (1993) 

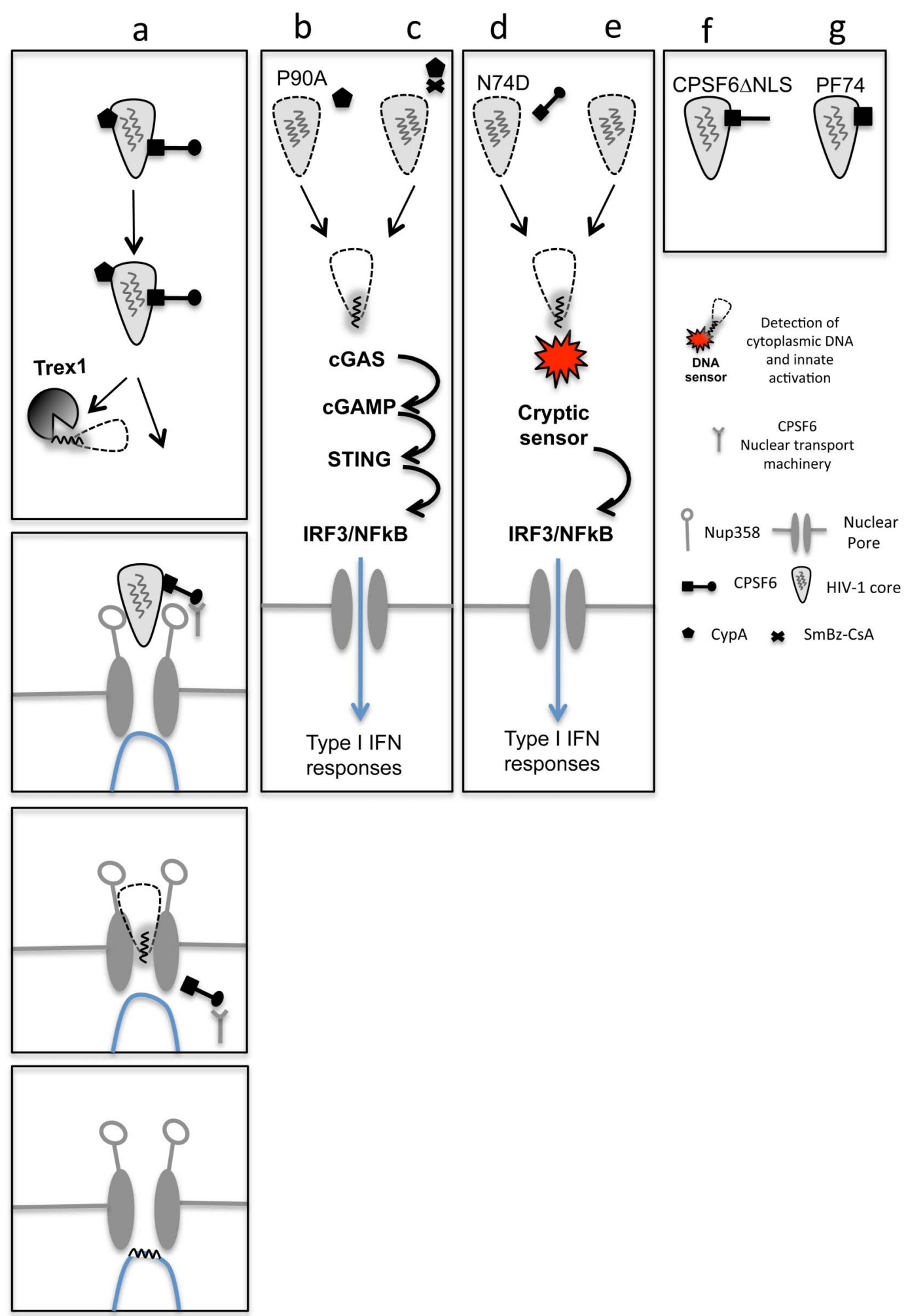

Extended Data Figure $1 \mid$ A model for HIV-1 core behaviour and innate sensing. a, Intact capsids recruit CypA and CPSF6 which direct the virus to the nucleus. CPSF6 interaction prevents premature DNA synthesis. Excess cytoplasmic DNA is degraded by TREX1. At the nuclear pore CPSF6 NLSdependent dissociation from the virus allows reverse transcription to proceed. Reverse-transcribed DNA crosses the nuclear membrane and integrates. b, $\mathbf{c}$, Disruption of CypA-CA interactions with either CA(P90A) mutation or cyclosporine treatment leads to detection of DNA reverse transcription product by cGAS initiating cGAMP production, STING activation, NF- $\mathrm{\kappa B} /$ IRF3 nuclear localization, type I interferon secretion and initiation of an antiviral state. d, e, Disruption of CPSF6-CA interactions by N74D CA mutation, or depletion of CPSF6, leads to activation of a cryptic innate DNA sensor which also activates NF- $\mathrm{B} / \mathrm{IRF} 3$ nuclear localization. f, Disruption of CPSF6 engagement with the nuclear transport machinery by mutating its NLS prevents reverse transcription because the CPSF6 does not dissociate from the capsid at the nuclear pore. (g) PF74 mimics CPSF6 by inserting a phenyl ring into a CA pocket in the same position as CPSF6 and also prevents reverse transcription. Like CPSF6 $\triangle$ NLS PF74 has no NLS and thus does not disengage from the core and therefore terminally prevents reverse transcription. 
a

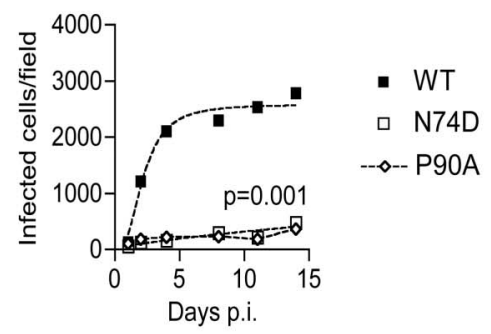

b

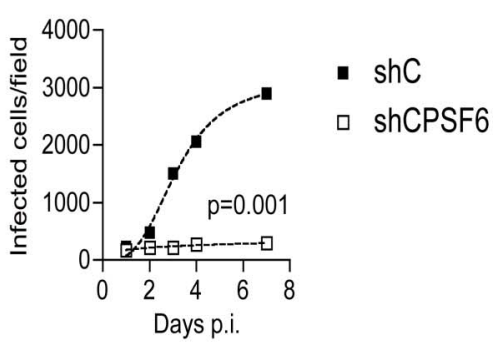

C

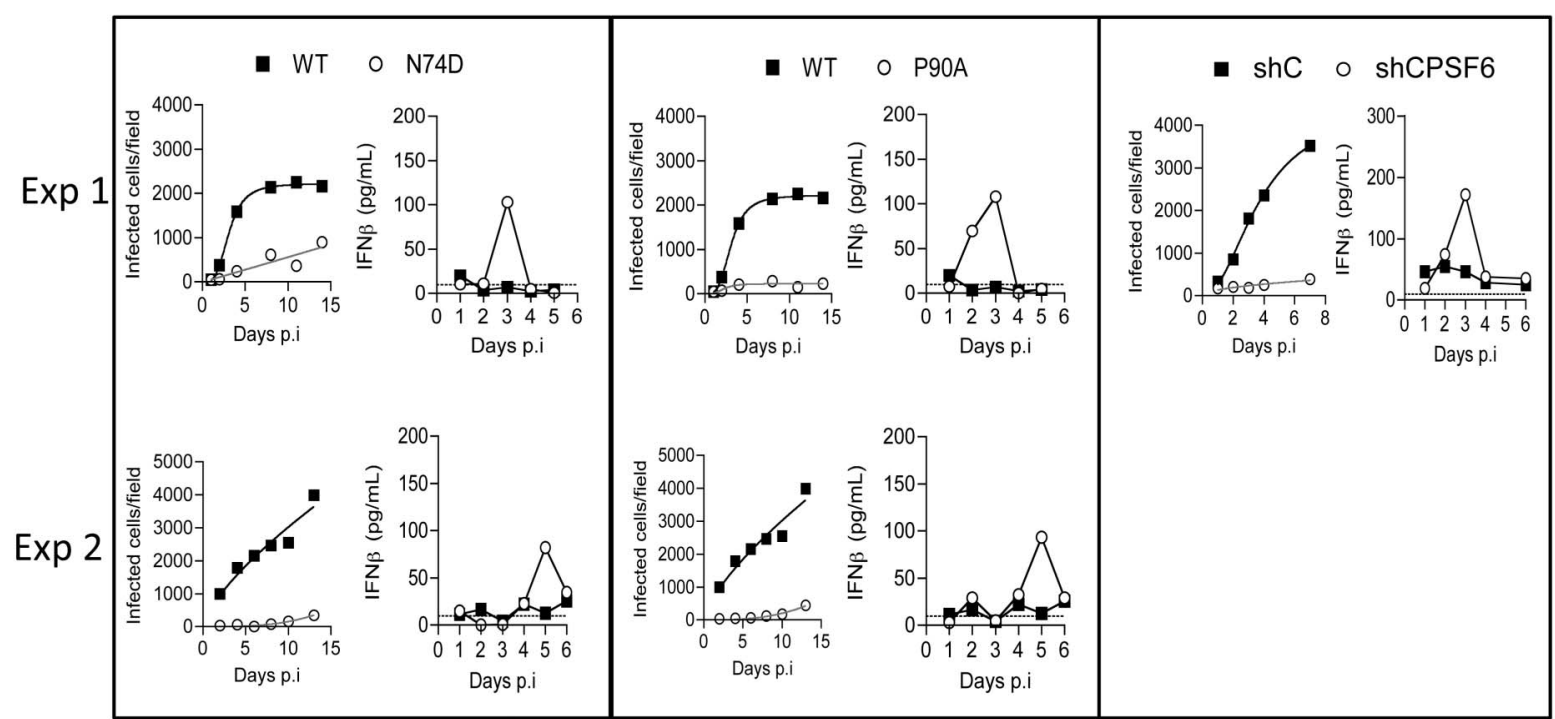

Extended Data Figure $2 \mid$ HIV-1 mutants CA N74D and P90A, or WT HIV1 on CPSF6 depletion, induce Type I IFN secretion in human macrophages that limits propagation. a, MDM were infected with HIV-1 WT, CA N74D or CA(P90A) at low multiplicity. Cells were stained for Gag p24 at specific time points after infection and infected colonies counted. b, MDM transduced to express shRNA targeting CPSF6, or a scrambled control hairpin, were infected with wild-type NL4.3 (Ba-L Env) at low multiplicity. Cells were stained for Gag p24 at specific time points after infection and infected colonies counted. (a-b) $P=0.001$, two-way ANOVA, for the effect of CA mutation or CPSF6 depletion. Data represent mean and nonlinear regression over time for 3 biological replicates. c, Individual experiments from two additional donors performed as experiments shown in Fig. 1a and b, and one additional donor performed as experiments in Fig. $2 \mathrm{c}$ and d. Each HIV-1 mutant is shown compared to wild-type virus data (WT) for comparison. 
a

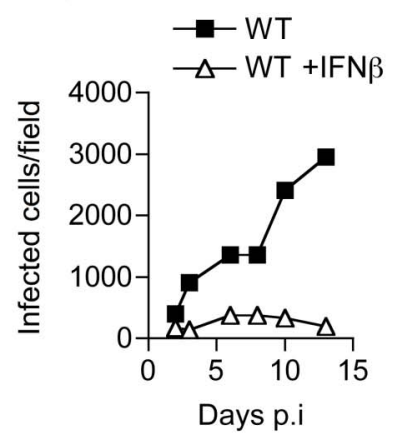

b

IFN- $\beta$

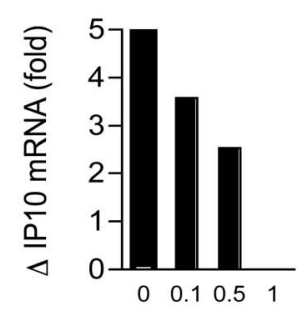

$\mu \mathrm{g} / \mathrm{ml} \mathrm{IFN} \alpha / \beta-\mathrm{R} A b$

C
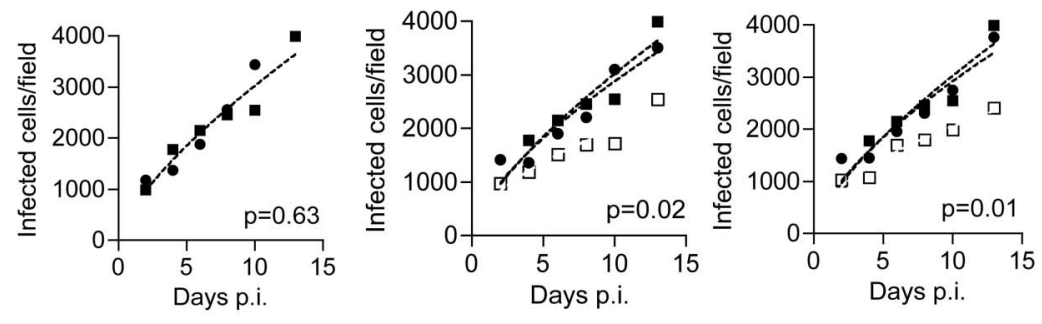

- WT

- WT

$W T+N 74 D$

- WT

- $W T+N 74 D+I F N \alpha / \beta-R A b$

- $W T+P 90 A$

- $W T+P 90 A+I F N \alpha / \beta-R A b$

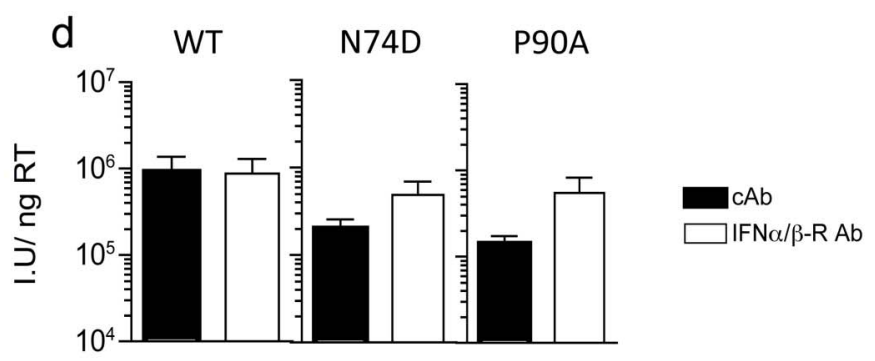

Extended Data Figure $3 \mid$ Suppression of HIV-1 by type 1 interferon and rescue of infectivity with anti-IFN receptor (IFNAR2) antibody. a, In order to demonstrate that IFN is able suppress wild type HIV-1 replication, MDM were pretreated with $1 \mathrm{ng} \mathrm{ml}^{-1}$ of recombinant IFN- $\beta$ for $2 \mathrm{~h}$ then infected with HIV-1 WT NL4.3 (BaL-Env) infection. Cells were stained for Gag p24 at specific time points post infection (p.i). b, In order to determine how much IFN- $\alpha / \beta$ receptor (IFNAR2) neutralizing antibody is required to neutralize an IFN response, MDM were pretreated with varying concentrations of antiIFNAR2 antibody for $2 \mathrm{~h}$ then stimulated with $1 \mathrm{ng} \mathrm{ml}^{-1}$ of recombinant IFN$\beta$ for $24 \mathrm{~h}$. IP10 gene expression levels were measured by qRT-PCR and normalized to GAPDH. Results are expressed as fold change of expression over untreated cells. $1 \mu \mathrm{g} \mathrm{ml}^{-1}$ of IFNAR2 antibody effectively neutralized
$1 \mathrm{ng} \mathrm{ml}^{-1}$ recombinant IFN- $\beta$, and this dose was used in subsequent experiments. c, MDM were infected with WT or WT and CA mutants at low multiplicity in the presence of anti-IFNAR2 antibody. Cells were stained for Gag at specific time points after infection and infected colonies counted ( $P$ values are given for two-way ANOVA for the effect of IFNAR blockade). Data represent mean and nonlinear regression of biological replicate experiments over time. d, Infectious titres of WT and CA mutant viruses were determined on MDM measured by assay of $\mathrm{p} 24$ positive cells $48 \mathrm{~h}$ post infection. Cells were infected in the presence of anti-IFNAR2 antibody or isotype control antibody (cAb). Titres are expressed as infectious units per nanogram of reverse transcriptase activity determined by ELISA. Mean \pm s.e.m. of titre determined at 3 doses (technical replicates). 
IP-10

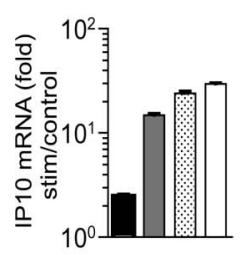

b
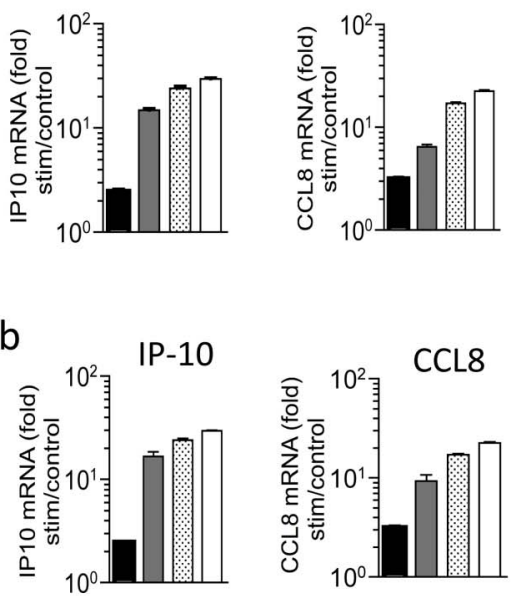

IFIT1

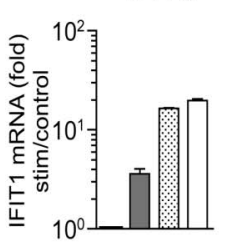

IFIT1

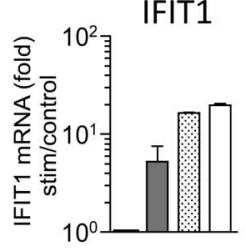

WT
$\square$ P90A
Polyl:C
$\square$ IFN- $\beta$

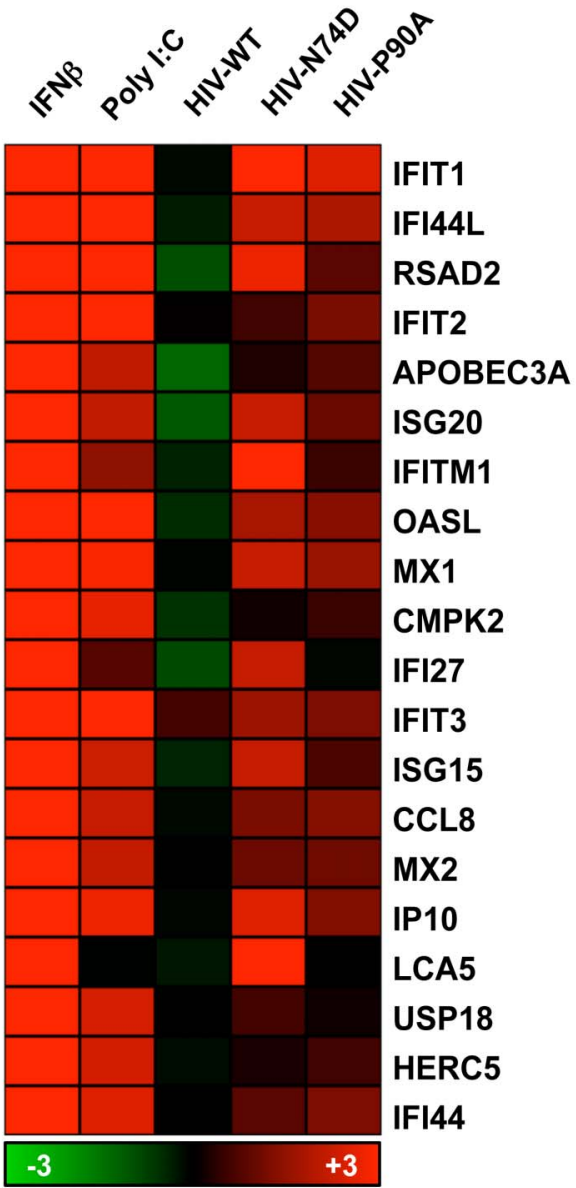

Fold change $\left(\log _{2}\right)$
Extended Data Figure $4 \mid$ Stimulation of gene expression by HIV-1 CA mutants in MDM. a, b, Selected IFN-stimulated genes significantly upregulated by HIV-1 CA mutant infection, as well as by IFN- $\beta$ and poly(I:C) measured at $24 \mathrm{~h}$ shown as fold change in expression (Stim/Control) measured by qRT-PCR and normalized to GAPDH mRNA levels. c, The same RNA samples as $\mathbf{a}, \mathbf{b}$ were subjected to expression array and are presented in an expression matrix illustrating fold change in gene expression. d, Upregulation of gene expression (mean $>2$ fold in 2 independent biological replicates) after infection of MDM by HIV-1 wild-type and mutants HIV-1 CA N74D and
HIV-1 CA(P90A) as shown. $24 \mathrm{~h}$ after infection (MOI 2), total RNA was isolated and subject to expression array, see methods. Results were subject to pathway analysis using the online bioinformatics tool- InnateDB (http:// www.innatedb.com). Type 1 IFN signalling was the most significantly overrepresented pathway with IFN- $\beta$, PolyIC and both HIV-1 mutants, but not WT virus, based on the Reactome database (http://www.reactome.org). The proportion of genes in each list that map to this pathway and the p-value following Benjamini-Hochberg correction for multiple biological replicates are indicated. 


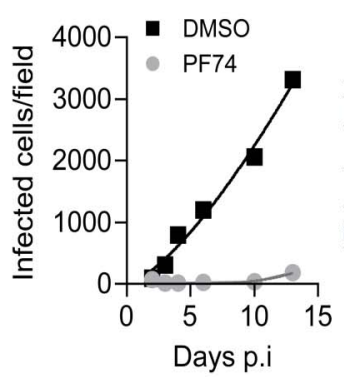

d

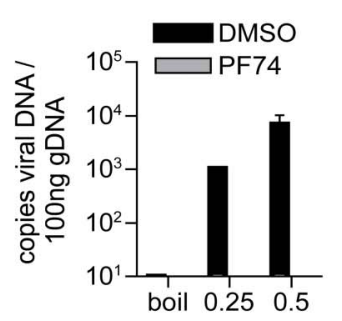

ng of NL4.3 (Ba-L Env) b

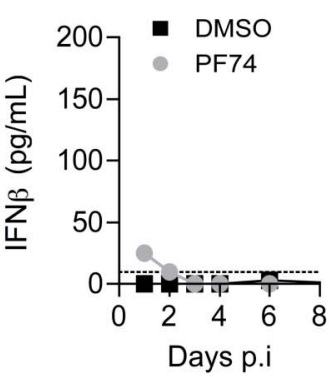

e

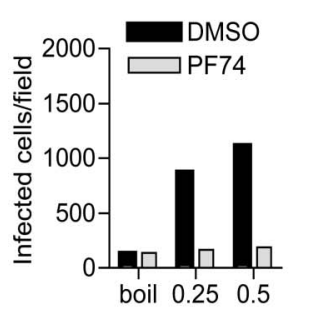

ng of NL4.3 (Ba-L Env)

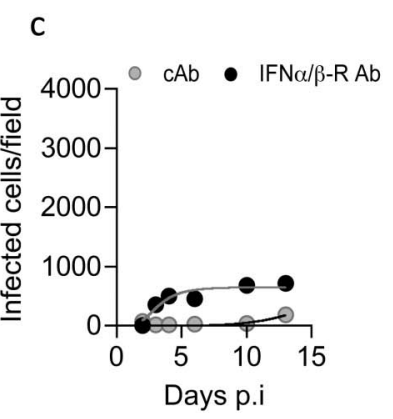

$f$

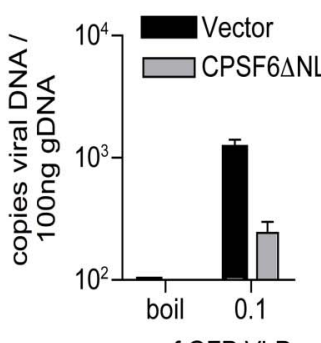

ng of GFP VLP g

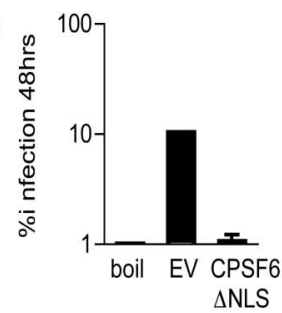

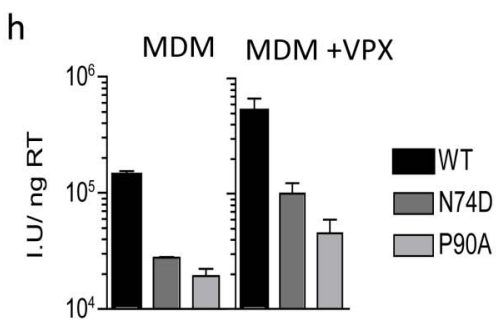

Extended Data Figure $5 \mid$ Inhibition of HIV-1 with PF74 in MDM does not trigger IFN production. a, Infection of human MDM with WT NL4.3 (Ba-L Env) in the presence or absence of $10 \mu \mathrm{M}$ PF74. Cells were stained for p24 at specific time points after infection and infected colonies counted. b, Supernatants collected from MDMs in a were assayed for soluble IFN- $\beta$ levels by ELISA. c, MDM were infected at low multiplicity with WT HIV-1 in the presence of PF74 and IFNAR2 antibody or isotype cAb. d, Measurement of HIV-1 late reverse transcription product (LRT) in MDM infected for $9 \mathrm{~h}$ with two concentrations of WT HIV-1, in the presence or absence of $10 \mu \mathrm{M}$ PF74. Cells infected with boiled virus served as a negative control for DNA contamination. e, A sample parallel to those in $\mathbf{d}$ was used to determine the number of infected cells by staining for $\mathrm{p} 2448 \mathrm{~h}$ post infection. f, Measurement of HIV-1 LRT product in HeLa cells that express CPSF6 $\triangle \mathrm{NLS}$ or empty vector (EV), infected for $9 \mathrm{~h}$ with VSV-G pseudotyped HIV-1 GFP. g, A parallel sample was used to determine the number of infected cells by flow cytometry $48 \mathrm{~h}$ post infection. $\mathbf{h}$. Infectious titres of WT and CA mutant viruses were determined on MDM in the presence and absence of SIVmac-VLPs encoding the SAMHD1 antagonist Vpx. Titres are expressed as infectious units per nanogram of reverse transcriptase activity determined by ELISA. Mean \pm s.e.m. of titre determined at 3 doses. Experiments represent 3 independent biological replicates. 
a

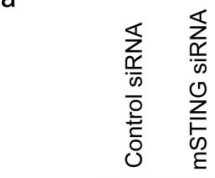

STING

$\beta$ - Actin

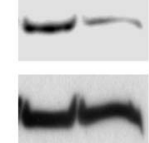

C $\quad$ Control siRnA

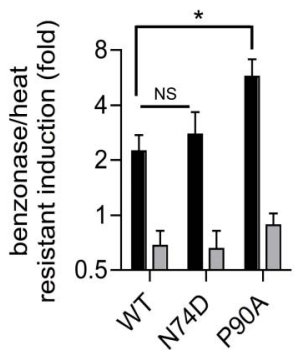

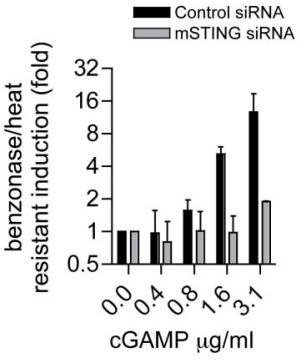

d

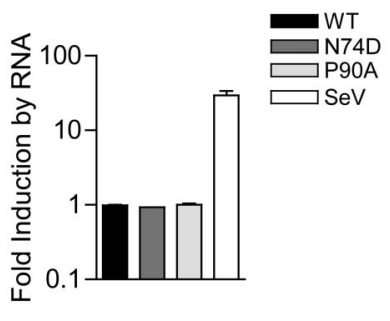

Extended Data Figure 6 | HIV-1 P90A CA mutant infected MDM contained a benzonase and heat resistant component that activated an interferon sensitive promoter. a, Immunoblot of extracts from L929 cells stably expressing Firefly Luciferase-driven by an interferon sensitive response element depleted of STING by siRNA or expressing control siRNA. b, L929 cells, control or STING depleted as in a, were treated with various concentrations of STING agonist cyclic GMP-AMP (cGAMP), and Luciferase activity was read after $16 \mathrm{~h}$. c, MDM were infected with HIV-1 wild-type (WT) or mutant HIV-1 CA N74D or HIV-1 CA(P90A) for $18 \mathrm{~h}$ (MOI of 2), total cell extracts were isolated and were heat and benzonase treated and were applied to L929 cells as in a and Luciferase activity measured at $16 \mathrm{~h}$ (Mean of $4 \pm$ s.e.m. of biological replicates). d, As c except RNA was extracted from the cell extracts and were transfected into $293 \mathrm{~T}$ cells to measure IFN- $\beta$ promoter driven Luciferase activity after $16 \mathrm{~h}$. Sendai virus infection served as positive control for immunostimulatory RNA (Mean \pm s.e.m. of biological replicates). c, * represents statistically significant difference between data sets $(P<0.05, t$-test), NS represents non-significant differences. 
a
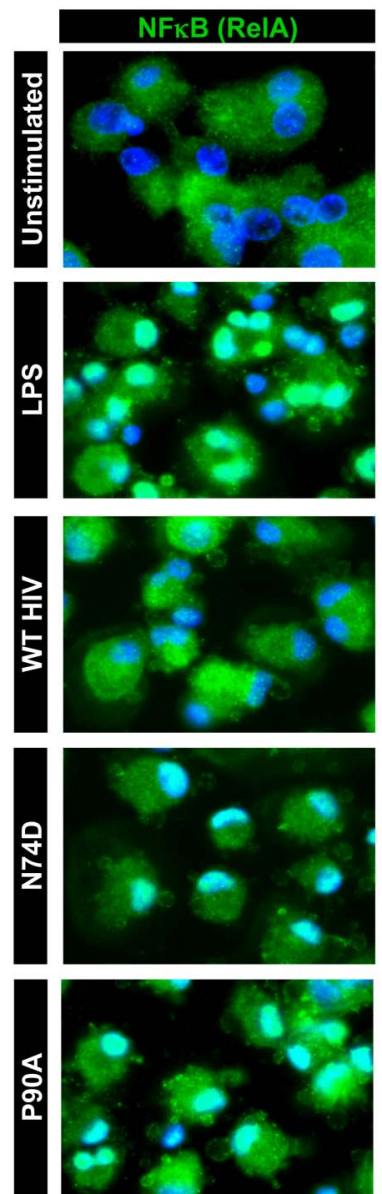
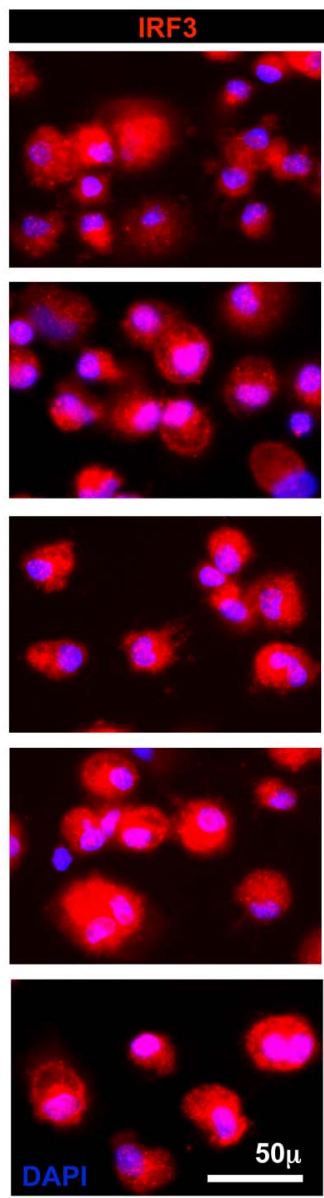
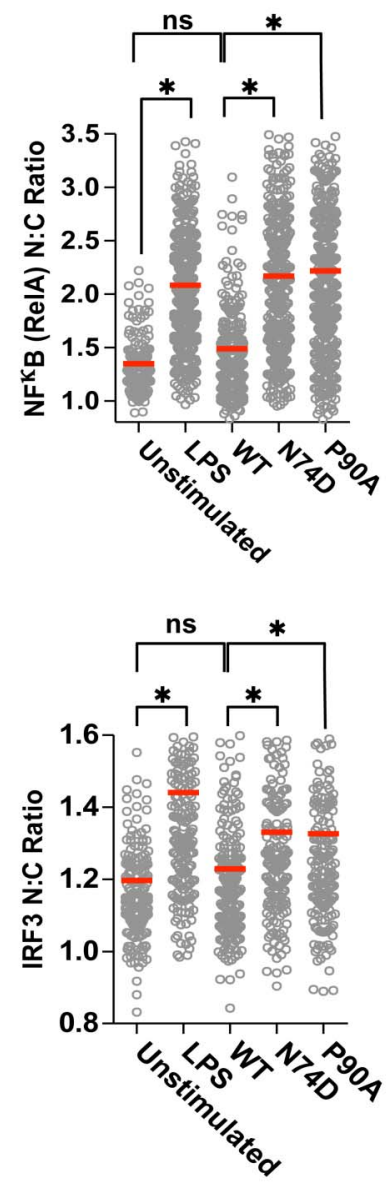

Extended Data Figure $7 \mid$ Nuclear translocation of NF-кB and IRF3 after HIV-1 capsid mutant infection in MDM. a, Confocal immunofluorescence microscopy was used to quantify nuclear translocation of NF- $\kappa$ B Rel A (green) and IRF3 (red) as a consequence of activation. Nuclear:cytoplasmic ratios of immunostaining were measured at single cell level by quantitation of NF- $\kappa \mathrm{B}$ or IRF3 signal intensities inside and outside the nucleus (blue DAPI) as described in methods. b, Data for 500 single cell measurements are shown for unstimulated MDM and MDM stimulated for $2 \mathrm{~h}$ with lipopolysaccharide (LPS) $\left(100 \mathrm{ng} \mathrm{ml}^{-1}\right.$ ), or infected with wild-type (WT) HIV-1 or N74D or P90A CA mutants. Red lines represent the mean of each data set. * represent statistically significant differences between data sets $(P<0.01, t$-test $)$, $n s$ represents non-significant differences $(P>0.05, t$-test). 
a

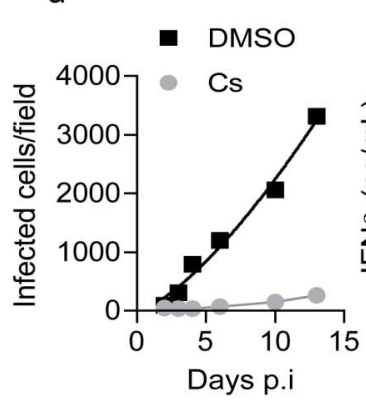

b

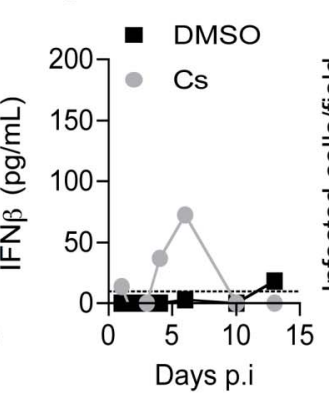

C

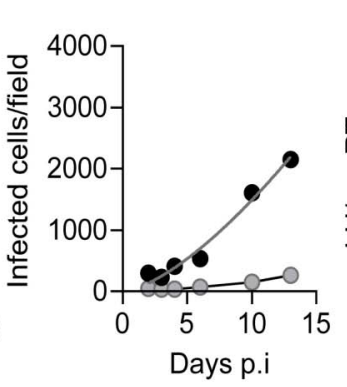

d

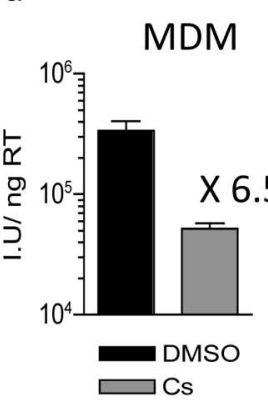

e

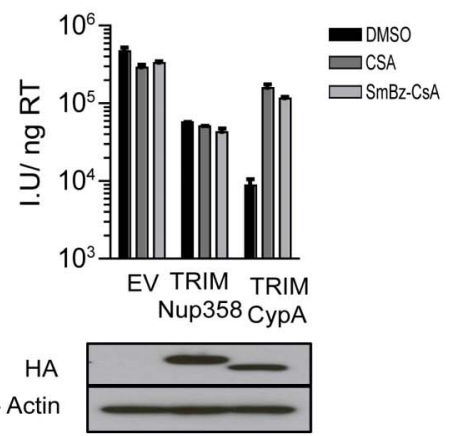

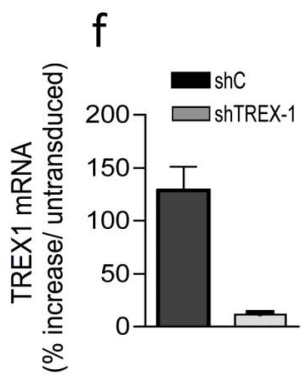

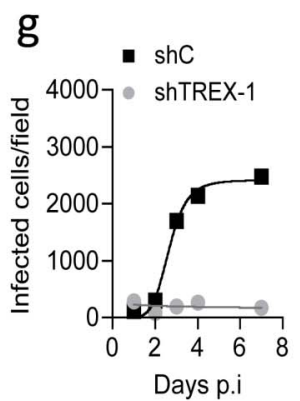

- $\mathrm{cAb} \bullet \mathrm{IFN} \alpha / \beta-\mathrm{R} A b$
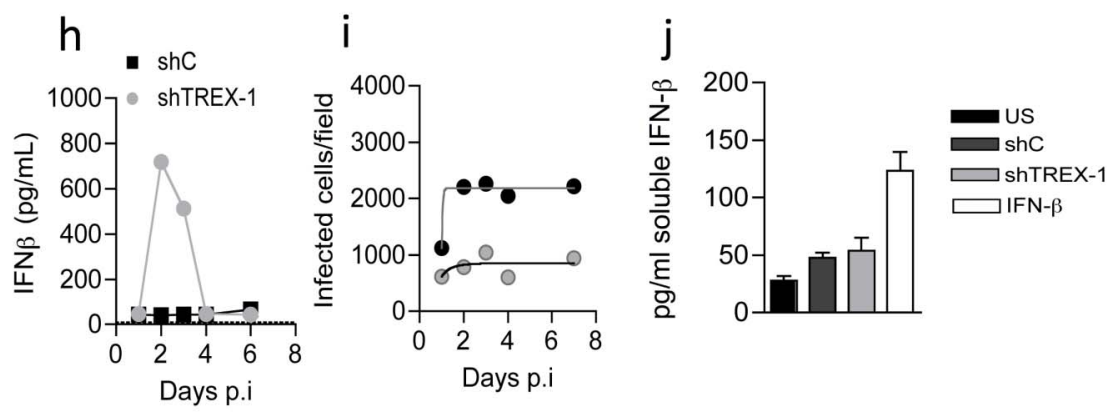

- $\mathrm{cAb} \bullet \mathrm{IFN} \alpha / \beta-\mathrm{R} A b$
Extended Data Figure $8 \mid$ Inhibition of HIV-1 with cyclosporine or by TREX depletion triggers IFN production. a, Infection of human MDM with WT NL4.3 (Ba-L Env) in the presence or absence of $5 \mu \mathrm{M}$ cyclosporine. Cells were stained for p24 at specific time points after infection and infected colonies counted. $\mathbf{b}$, Supernatants isolated from MDMs in a were assayed for soluble IFN- $\beta$ levels by ELISA. c, MDM were infected at low multiplicity with WT HIV in the presence of cyclosporine and IFNAR2 antibody or isotype cAb. d, Infectious titre of WT HIV was determined on MDM in the presence of DMSO or cyclosporine at $48 \mathrm{~h}$ post infection. The data are presented as infectious units (iu) per nanogram (ng) of reverse transcriptase (RT) measured by ELISA. e, To confirm that SmBz-CsA inhibits recruitment of cyclophilin A (CypA), but not Nup358 Cyp, to HIV-1 CA, we used the TRIMCyp restriction assay. HIV-1 GFP vector titer on CRFK cells expressing empty vector (EV), HA-tagged owl monkey TRIMCyp RBCC domain fused to human CypA (TRIMCypA) or human Nup358Cyp (TRIMNup358) in either DMSO, or $5 \mu \mathrm{M}$ cyclosporine or $10 \mu \mathrm{M} \mathrm{SmBz}$-CsA. Protein levels were measured by immunoblot detecting the HA tag with $\beta$-Actin as a loading control. In this assay both cyclosporine and SmBz-CsA inhibited CypA recruitment to CA and rescued VSV-G pseudotyped HIV-1 GFP infectivity from restriction by TRIMCypA. However, neither drug rescued HIV-1 infectivity from TRIMNup358, confirming cyclosporine specificity for CypA and not Nup358. f, TREX-1 expression was determined in MDM expressing TREX-1 specific shRNA or control shRNA by qRTPCR, normalized to GAPDH at the time of HIV-1 infection in g. g, Cells were stained for p24 at specific time points after WT HIV-1 infection of TREX-1 depleted and control shRNA expressing MDM and infected colonies counted. $\mathbf{h}$, Supernatants isolated from MDM in $\mathbf{g}$ were assayed for soluble IFN- $\beta$ by ELISA. $\mathbf{i}$, Infection of TREX-1 depleted MDM with WT HIV-1 at low multiplicity in the presence of either IFNAR2 antibody or isotype cAb. Cells were stained for p24 at specific time points after infection and infected colonies counted. $\mathbf{j}$, Hairpin transduced MDM were assayed for the production of soluble IFN- $\beta$ levels before HIV-1 infection. Data are representative of 3 independent biological replicates. For HIV-1 replication assays in MDM data points and nonlinear regression lines over time are shown. 

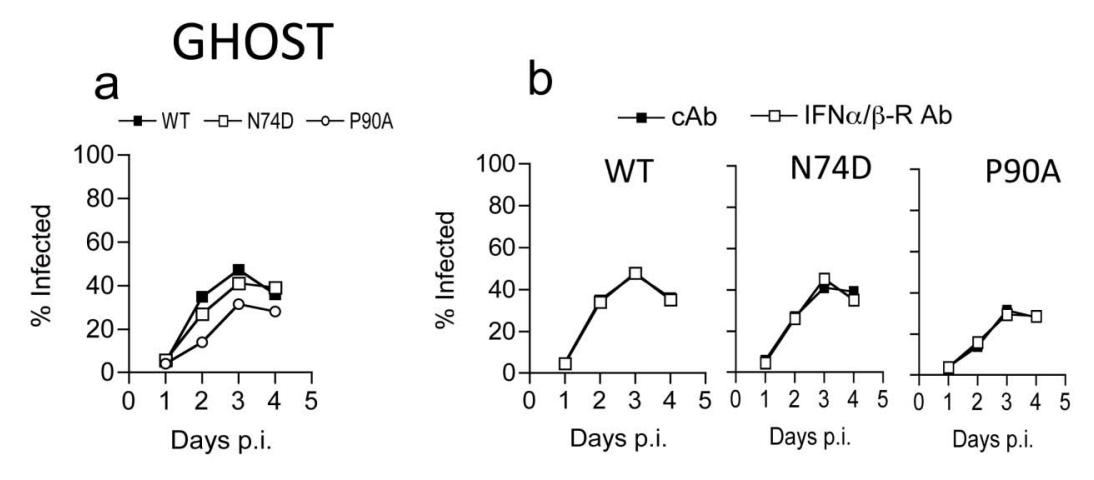

C
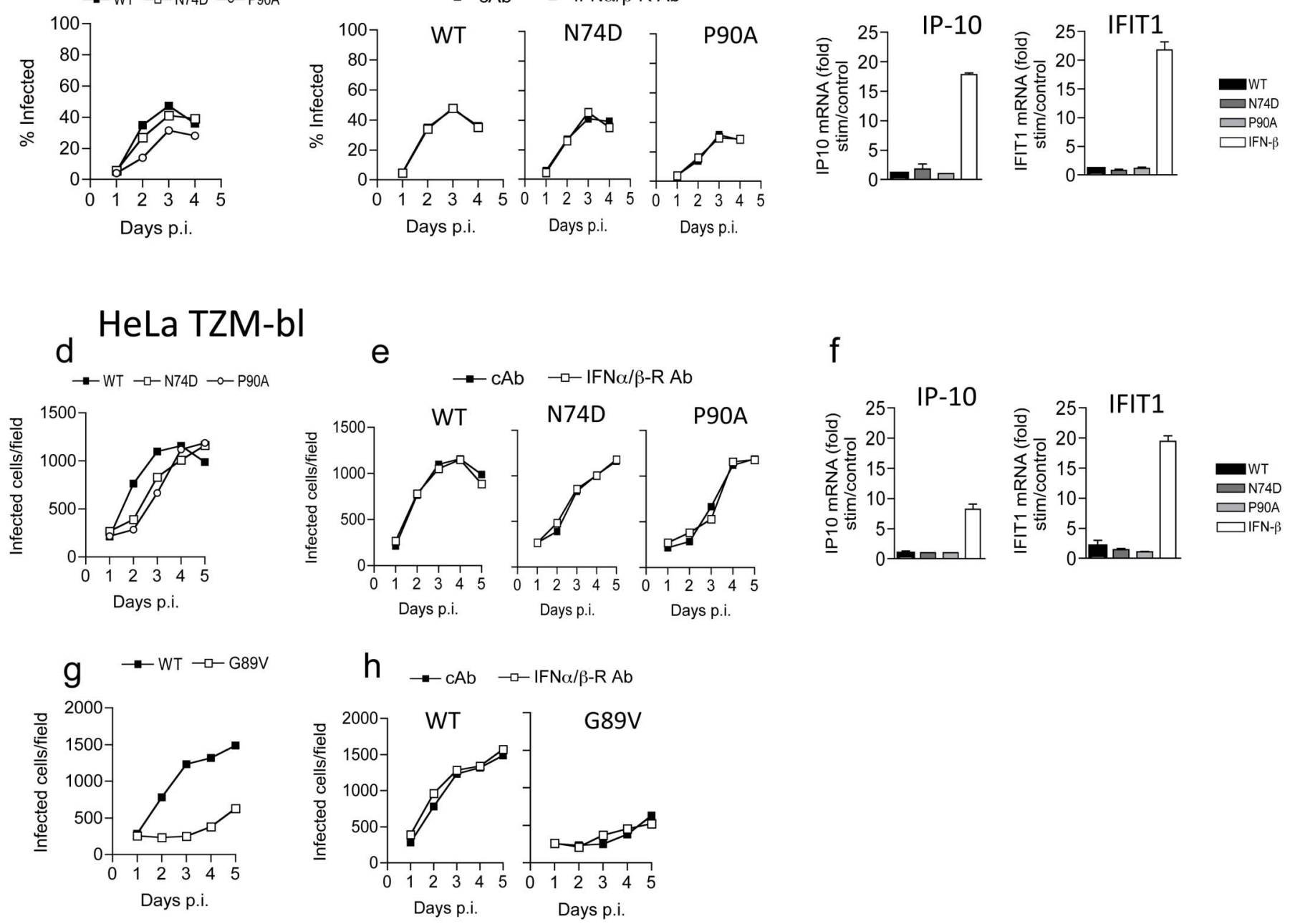

Extended Data Figure 9 $\mid$ HIV-1 CA mutants replicate without triggering IFN production in cell lines. GHOST $(\mathbf{a}-\mathbf{c})$ or HeLa TZM bl $(\mathbf{d}-\mathbf{f})$ indicator cell lines were infected with WT NL4.3 (Ba-L Env) or NL4.3 (Ba-L Env) bearing CA mutations N74D or P90A at low multiplicity (0.04). Replication was monitored by GFP expression (GHOST) or staining LacZ positive cells (HeLa $\mathrm{TZM} \mathrm{bl).} \mathrm{Both} \mathrm{mutants} \mathrm{replicated} \mathrm{well,} \mathrm{slightly} \mathrm{behind} \mathrm{wild-type} \mathrm{virus.}$ Replication in GHOST (b) or HeLa TZM bl (e) was performed in the presence of IFNAR2 antibody or isotype cAb. Neither antibody had any effect on WT or mutant HIV-1 replication in GHOST or HeLa TZM bl indicator cell lines. $\mathbf{c}, \mathbf{f}$, Induction of ISGs IP10 and IFIT1 expression were measured by

quantitative RT PCR after high multiplicity infection (MOI 2) by WT or CA mutant HIV-1 on GHOST (c) or HeLa TZM bl (f). $10 \mathrm{ng} \mathrm{ml}^{-1}$ of IFN- $\beta$ treatment acted as a positive control. ISG expression levels were normalized to GAPDH and are expressed as fold change in expression over unstimulated cells (Mean of 3 replicates \pm s.e.m.). Neither WT or CA mutant HIV-1 induced ISG expression in either cell line. g, HeLa TZM bl were infected WT or CA G89V at low multiplicity (0.04). Replication was monitored by staining LacZ positive cells. h, HIV-1 CA G89V replication in HeLa TZM bl was defective and not rescued by anti-IFNAR2 or isotype control antibodies. All results are representative of 2 biological replicates. 
Extended Data Table 1 | Structure data collection and refinement statistics

Extended Data Table 1 Structure data collection and refinement statistics

\begin{tabular}{ll}
\hline & CypA:SmBz-CsA \\
\hline $\begin{array}{l}\text { Data collection } \\
\text { Space group } \\
\text { Cell dimensions }\end{array}$ & 1222 \\
$\quad a, b, c(\AA)$ & $54.18,64.60,80.07$ \\
$\quad a, b, g\left({ }^{\circ}\right)$ & $90.00,90.00,90.00$ \\
Resolution $(\AA)$ & $32.30-1.67(1.76-1.67)^{*}$ \\
$R_{\text {merae }}$ & $0.061(0.562)$ \\
I/sI & $17.1(2.9)$ \\
Completeness $(\%)$ & $98.2(91.1)$ \\
Redundancy & $6.0(5.8)$ \\
& \\
Refinement & \\
Resolution $(\AA)$ & 1.67 \\
No. reflections & 15648 \\
$R_{\text {workl }} R_{\text {free }}$ & $0.172 / 0.218$ \\
No. atoms & \\
$\quad$ Protein & 1250 \\
Ligand/ion & 97 \\
Water & 184 \\
B-factors & \\
Protein & 19.485 \\
Ligand/ion & 25.523 \\
Water & 30.397 \\
R.m.s deviations & \\
$\quad$ Bond lengths $(\AA)$ & 0.005 \\
Bond angles $\left({ }^{\circ}\right)$ & 1.082 \\
\hline *Highest resolution shell is shown in parenthesis. & \\
&
\end{tabular}

*Highest resolution shell is shown in parenthesis. 